\title{
The Fuzzy System with a Family of Implication Operators $L-\lambda-G$ Based on Triple I Algorithm
}

\author{
Jiayin Peng \\ Department of Mathematics, Neijiang Teachers College, Neijiang, Sichuan 641112, P. R. China
}

\begin{abstract}
Firstly, we establish a formula of FMP problems by Triple I algorithm based on a family of implication operators $L-\lambda-G$. Secondly, we discuss fuzzy systems based on Triple I algorithm and their response ability. The results show that the fuzzy system with a family of implication operators $L-\lambda-G$ has only step output property rather than universal approximation; Finally, we expose its significance of probability and give its probability distribution. It seems that it plays a role of the kernel of system in some fuzzy system.
\end{abstract}

Keywords: Fuzzy system, Family of implication operators $L-\lambda-G$, Triple I algorithm, Conditional mathematical expectation, Probability distribution

\section{Introduction}

In 1973, L.A.Zadeh proposed the ideal of fuzzy inference and gave the famous CRI algorithm [1]. Fuzzy technology associated to fuzzy control is widely applied to the various areas such as industry, agriculture and science research. Due to it, much economic benefit has been obtained. Wang [2] gave the famous total implication Triple I algorithm with respect to fuzzy inference in 1999. He generalized the famous CRI algorithm and made it into the frame of Fuzzy Logic. It was pointed out that fuzzy system based on Triple I algorithm was a kind of interpolation in nature in [3]. Fuzzy systems based on CRI algorithm and Triple I Algorithm were investigated in [4] and [5], respectively. It was pointed out that response ability of constructing fuzzy system by the distinct implication operators may be of much difference. Some fuzzy systems with implication operators have universal approximation. But some fuzzy systems with implication operators have only step output property rather than universal approximation. Thus they can not be applied to the fuzzy control systems. Wang [6] and Wu [7] independently proposed implication operators with parameters. Zhang [8] proposed a family of implication operators $L-\lambda-G$. Is it true that a constructed fuzzy system with implication operators and parameters based on Triple I algorithm has universal approximation? We normally consider the problem. Therefore, in the paper we study the response ability and probability representation of a constructed fuzzy system with implication operators $L-\lambda-G$ and parameters based on Triple I algorithm.

\section{Total implication Triple I algorithm concerned about FMP problems of a family of implication operators $L-\lambda-G$}

In reference [8], fuzzy implication operator $R(x, y)=x \rightarrow_{\lambda} y$ with parameter $\lambda \in[0,1]$ is defined by

$x \rightarrow_{\lambda} y=\left\{\begin{array}{cc}1, & x \leq y \\ \lambda(1-x)+y, & x>y\end{array} \quad x, y \in[0,1]\right.$.

To be specific, if $\lambda=1,0$ then implication operators $R(x, y)$ become Lukasiewiez implication operator $R_{L u}$ and Godel implication operator $R_{G}$, respectively. When $\lambda$ varies in the interval $[0,1]$, all operators $R(x, y)$ defined in representation (1) are called a family of implication operators $R_{L-\lambda-G}$.

Firstly, the principle concerned about FMP problem by total implication Triple I based on fuzzy inference is given in the following:

Let the notions $X$ and $Y$ denote nonempty sets. $\mathcal{F}(X)$ and $\mathcal{F}(Y)$ denote the corresponding collections of all fuzzy subsets of $X$ and $Y$. If conditions $A, A^{*} \in \mathcal{F}(X), B \in \mathcal{F}(Y)$ are given, we wish to looks for a minimum fuzzy set $B^{*} \in \mathcal{F}(Y)$ such that

$$
(A(x) \rightarrow B(y)) \rightarrow\left(A^{*}(x) \rightarrow B^{*}(y)\right)
$$


has maximal value for any $x \in X$ and $y \in Y$.

The result describing the support solutions of Triple I concerned about FMP problems of a family of implication operators $L-\lambda-G$ is stated as follows.

Theorem 2.1 The support solution $B^{*}$ of Triple I concerned about FMP problems of a family of implication operators $L-\lambda-G$ satisfies the following formula

$$
\begin{aligned}
B^{*}(y)= & \sup _{x \in X}\left\{A^{*}(x) \wedge[(R(A(x), B(y))\right. \\
& \left.\left.\left.-\lambda\left(1-A^{*}(x)\right)\right) \vee 0\right]\right\}, y \in Y .
\end{aligned}
$$

Proof. For an arbitrary $y \in Y$ and $C \in \mathcal{F}(Y)$ satisfying $C(y) \geq B^{*}(y)$, formula (2) has a maximal value 1 . In fact, for any $x \in X$, we obtain from $C(y) \geq B^{*}(y)$ and formula (3)

$$
\begin{aligned}
C(y) \geq & A^{*}(x) \wedge[(R(A(x), B(y)) \\
& \left.\left.-\lambda\left(1-A^{*}(x)\right)\right) \vee 0\right] .
\end{aligned}
$$

Note that the properties of the family of implication operators $L-\lambda-G$. They are non-increasing in the first argument and non-decreasing in the second argument. Thus two cases are considered as follows

(i) If $C(y) \geq A^{*}(x)$, then $(A(x) \rightarrow B(y)) \rightarrow$ $\left(A^{*}(x) \rightarrow C(y)\right) \geq(A(x) \rightarrow B(y)) \rightarrow\left(A^{*}(x) \rightarrow\right.$ $\left.A^{*}(x)\right)=(A(x) \rightarrow B(y)) \rightarrow 1 \equiv 1$.

(ii) If $C(y)<A^{*}(x)$, then it yields that $C(y) \geq R(A(x), B(y))-\lambda\left(1-A^{*}(x)\right.$ from formula (4). Therefore

$$
\begin{aligned}
& (A(x) \rightarrow B(y)) \rightarrow\left(A^{*}(x) \rightarrow C(y)\right) \\
=\quad & R(A(x), B(y)) \rightarrow\left(\lambda\left(1-A^{*}(x)\right)+C(y)\right) \equiv 1 .
\end{aligned}
$$

On the other hand, If there exists $y_{0} \in Y$ satisfying $D\left(y_{0}\right)<B^{*}\left(y_{0}\right)$ and $D \in \mathcal{F}(Y)$, then formula (2) can not reach the maximum 1. Actually, from $D\left(y_{0}\right)<B^{*}\left(y_{0}\right)$ and formula (3), there exists $x_{0} \in X$ such that

$$
\begin{aligned}
D\left(y_{0}\right)< & A^{*}\left(x_{0}\right) \wedge\left[\left(R\left(A\left(x_{0}\right), B\left(y_{0}\right)\right)\right.\right. \\
& \left.\left.-\lambda\left(1-A^{*}\left(x_{0}\right)\right)\right) \vee 0\right] .
\end{aligned}
$$

If $R\left(A\left(x_{0}\right), B\left(y_{0}\right)\right)-\lambda\left(1-A^{*}\left(x_{0}\right)\right) \leq 0$, there is no $D \in \mathcal{F}(Y)$ that satisfies $D\left(y_{0}\right)<B^{*}\left(y_{0}\right)$.

If $R\left(A\left(x_{0}\right), B\left(y_{0}\right)\right)-\lambda\left(1-A^{*}\left(x_{0}\right)\right)>0$, we get from $(5)$

$$
\begin{aligned}
& \left(A\left(x_{0}\right) \rightarrow B\left(y_{0}\right)\right) \rightarrow\left(A^{*}\left(x_{0}\right) \rightarrow D\left(y_{0}\right)\right) \\
=\quad & \left(A\left(x_{0}\right) \rightarrow B\left(y_{0}\right)\right) \rightarrow\left(\lambda\left(1-A^{*}\left(x_{0}\right)\right)+D\left(y_{0}\right)\right) \\
=\quad & \lambda\left(1-R\left(A\left(x_{0}\right), B\left(y_{0}\right)\right)\right)+\lambda\left(1-A^{*}\left(x_{0}\right)\right) \\
& +D\left(y_{0}\right) \\
\leq \quad & 1-R\left(A\left(x_{0}\right), B\left(y_{0}\right)+\lambda\left(1-A^{*}\left(x_{0}\right)\right)\right. \\
& \left.+D\left(y_{0}\right)\right)<1 .
\end{aligned}
$$

Putting together the results above, we claim that $B^{*}$ is a minimal fuzzy set of $\mathcal{F}(Y)$ satisfying (2).

\section{Fuzzy systems with a fam- ily of implication operators $L-\lambda-G$ based on Triple I algorithm and their response ability}

Suppose that the input universes $X$ and the output universe $Y$ are limited to one-dimensional real space $\mathbb{R}$, i.e., $X, Y \in \mathcal{B}^{1}$, where $\mathcal{B}^{1}$ denotes onedimensional Borel $\sigma$ - field. Pick $\mathcal{A}=\left\{A_{i} \mid 1 \leq i \leq\right.$ $n\} \subset \mathcal{F}(X)$ and $\mathcal{B}=\left\{B_{i} \mid 1 \leq i \leq n\right\} \subset \overline{\mathcal{F}}(Y)$. $\mathcal{A}$ and $\mathcal{B}$ are respectively collections of some fuzzy subsets of $X$ and $Y$. Fuzzy sets $A_{i}$ and $B_{i}$ are called language value. $\mathcal{A}$ and $\mathcal{B}$ are regarded as language variable, and they get the values of $A_{i}$ and $B_{i}$ in themselves. Thus $n$ - pieces of fuzzy inference rules can be formed as follows

$$
\text { if } x \text { is } A_{i} \text {, then } y \text { is } B_{i}, i=1,2, \cdots, n \text {. }
$$

$x \in X$ and $y \in Y$ are called basic variable with respect to language variable $\mathcal{A}$ and $\mathcal{B}$. Hence we obtain the following relation

$s^{*}: \mathcal{A} \rightarrow \mathcal{B}, A_{i} \mapsto s^{*}\left(A_{i}\right) \triangleq B_{i}, i=1,2, \cdots, n$.

The fuzzy relationship $R_{i} \in \mathcal{F}(X \times Y)$ formed by the $i$-th rule in (2) is also called truth universe. It is defined by $L-\lambda-G$ implication operator $R_{L-\lambda-G}$. Namely, $R_{i}(x, y) \triangleq R_{L-\lambda-G}\left(A_{i}(x), B_{i}(y)\right)$. These $n$ rules are linked by OR (it corresponds to union of sets). Thus the whole inference rules is represented by $\tilde{R} \triangleq \bigcup_{i=1}^{n} R_{i}$, i.e.,

$$
\begin{aligned}
\tilde{R}(x, y) & =\bigvee_{i=1}^{n} R_{i}(x, y) \\
& =\bigvee_{i=1}^{n} R_{L-\lambda-G}\left(A_{i}(x), B_{i}(y)\right)
\end{aligned}
$$

The process of forming the whole inference rules by these parts of inference rules $R_{i}$ is called rule synthesis.

For an arbitrary given $A \in \mathcal{F}(X)$, an inference result $B \in \mathcal{F}(Y)$ is obtained from the whole inference rules $\tilde{R}$. This means that function relation $s^{* *}$ is constructed, i.e.

$$
s^{* *}: \mathcal{F}(Y) \rightarrow \mathcal{F}(Y), A \mapsto s^{* *}(A)=B,
$$


where $B$ is a minimal fuzzy set such that $\tilde{R}(x, y) \rightarrow$ $(A(x) \rightarrow B(y))$ has a maximal possible value for all $x \in X$ and $y \in Y$. Hence, according to Theorem 2.1, we have

$$
\begin{aligned}
B(y)= & \sup _{x \in X}\left\{A ( x ) \wedge \left[\left(\bigvee_{i=1}^{n} R_{L-\lambda-G}\left(A_{i}(x), B_{i}(y)\right)\right.\right.\right. \\
& -\lambda(1-A(x))) \vee 0]\}, y \in Y
\end{aligned}
$$

For an arbitrary given $x^{\prime} \in X$, in order to use (10), we set $x_{0}=x^{\prime}$, fuzzify $x^{\prime}$ and usually adopt a fuzzy set $A^{\prime}$ with single point:

$$
A^{\prime}(x)=\left\{\begin{array}{l}
1, x=x^{\prime} \\
0, x \neq x^{\prime}
\end{array}\right.
$$

Substituting it to (10), we obtain an inference result $B^{\prime} \in \mathcal{F}(Y)$

$$
B^{\prime}(y)=\bigvee_{i=1}^{n} R_{L-\lambda-G}\left(A_{i}\left(x^{\prime}\right), B_{i}(y)\right), y \in Y .
$$

Since $B^{\prime}$ is a fuzzy set, a defuzzify-method is used to obtain the accurate scalar $y^{\prime} \in Y$. If $\int_{Y}|y| B^{\prime}(y) d y<+\infty$ and $0<\int_{Y} B^{\prime}(y) d y<+\infty$, then center of gravity defuzzifier is often used to obtain $y^{\prime}$, i.e.

$$
y^{\prime}=\frac{\int_{Y} y B^{\prime}(y) d y}{\int_{Y} B^{\prime}(y) d y} .
$$

Note $x^{\prime}$ and $y^{\prime}$ are expressed in order to deduce formula (13) clearly. Now we substitute $x^{\prime}$ for $x$ and substitute $y^{\prime}$ for $\bar{s}(x)$. Then we can get

$$
\begin{aligned}
\bar{s}: \quad X & \rightarrow Y \\
x & \mapsto \bar{s}(x) \\
& \triangleq \int_{Y} y B^{\prime}(y) d y / \int_{Y} B^{\prime}(y) d y .
\end{aligned}
$$

Suppose that $\mathcal{A}$ and $\mathcal{B}$ are fuzzy divisions of $X$ and $Y$, respectively. There is no lose of generality in assuming that $X=[a, b]$ and $Y=[c, d]$ are both real intervals, which are divided by means of $a<$ $x_{1}<x_{2}<\cdots<x_{n}<b, c<y_{1}<y_{2}<\cdots<y_{n}<$ $d$, where $x_{i}, y_{i}$ are respectively peak points of $A_{i}$, $B_{i}$. If $X$ and $Y$ are general measurable sets, the following conclusion holds

Theorem 3.1 Under the assumptions above, the fuzzy system with a family of implication operators $L-\lambda-G$ based on Triple I algorithm approximates to a step function

$$
F(x)=\frac{1}{y_{n}-c} \sum_{i=1}^{n} h_{i} y_{i}=\text { const } .
$$

Proof. Set $h_{1}=y_{1}-c, h_{i}=y_{i}-y_{i-1}(i=$ $2,3, \cdots, n)$. Because $A$ and $B$ denote respectively fuzzy divisions of $X$ and $Y$, They have Kronecher property: $A_{i}\left(x_{j}\right)=\delta_{i j}=B_{i}\left(y_{j}\right)$. From (15) and the definition of definite integral, we have

$$
\begin{aligned}
y^{\prime} & =\frac{\int_{c}^{d} y B^{\prime}(y) d y}{\int_{c}^{d} B^{\prime}(y) d y} \approx \frac{\sum_{i=1}^{n} y_{i} B^{\prime}\left(y_{i}\right) h_{i}}{\sum_{i=1}^{n} B^{\prime}\left(y_{i}\right) h_{i}} \\
& =\frac{\sum_{i=1}^{n} h_{i}\left[\bigvee_{k=1}^{n} R_{L-\lambda-G}\left(A_{k}\left(x^{\prime}\right), B_{k}\left(y_{i}\right)\right)\right] y_{i}}{\sum_{i=1}^{n} h_{i}\left[\bigvee_{k=1}^{n} R_{L-\lambda-G}\left(A_{k}\left(x^{\prime}\right), B_{k}\left(y_{i}\right)\right)\right]} \\
& =\frac{1}{y_{n}-c} \sum_{i=1}^{n} h_{i} y_{i}=\text { const. }
\end{aligned}
$$

If $F(x)=\frac{1}{y_{n}-c} \sum_{i=1}^{n} h_{i} y_{i}$, then the theorem is proved.

Remark 1 Since $1 /\left(y_{n}-c\right)$ is a constant, $F(x)$ is also. Namely, the interpolation function of the fuzzy system is a step function, i.e. a function with constant. Obviously, it has not interpolation property. From the aspect of function approximation, $F(x)$ is trivial. Therefore the fuzzy system can almost be unapplied in the practical fuzzy control system.

Suppose that the input universes $X, Y$ and the output universe $Z$ is limited to one-dimensional space $\mathbb{R}$, i.e., $X, Y, Z \in \mathcal{B}^{1}$, where $\mathcal{B}^{1}$ denotes onedimensional Borel $\sigma$ - field. $\mathcal{A}=\left\{A_{i} \mid 1 \leq i \leq n\right\} \subset$ $\mathcal{F}(X), \mathcal{B}=\left\{B_{i} \mid 1 \leq i \leq n\right\} \subset \mathcal{F}(Y), \mathcal{C}=\left\{C_{i} \mid 1 \leq\right.$ $i \leq n\} \subset \mathcal{F}(Z)$ are respectively fuzzy divisions of $X, Y$ and $Z$. Similarly, we give the $\operatorname{sign} Z=[e, f]$ as shown above. Suppose that $z_{i}$ is a peak point of $C_{i}$ and satisfies $e<z_{1}<z_{2}<\cdots<z_{n}<f$. And assume that $C_{i}$ is a integrable function.

Theorem 3.2 Under the assumptions above, the fuzzy system with a family of double input and single output implication operators $L-\lambda-G$ based on Triple I algorithm approximates to a step function

$$
F(x, y)=\sum_{i=1}^{n} \frac{h_{i}}{z_{n}-e} z_{i}=\text { const }
$$

Proof. Set $\mathcal{A}, \mathcal{B}, \mathcal{C}$ are language variables. Hence $n$-pieces of fuzzy inference rules can be formed as follows

$$
\begin{aligned}
& \text { if } x \text { is } A_{i} \text { and } y \text { is } B_{i}, \\
& \text { then } z \text { is } C_{i}, i=1,2, \cdots, n \text {. }
\end{aligned}
$$

The whole truth universe $\tilde{R}=\bigcup_{i=1}^{n} R_{i}$ formed by the $n$ pieces of rules is determined by $L-\lambda-G$ 
implication operator $R_{L-\lambda-G}$. Namely,

$$
\begin{aligned}
\tilde{R}(x, y, z) & =\bigvee_{i=1}^{n} R_{i}(x, y, z) \\
& =\bigvee_{i=1}^{n} R_{L-\lambda-G}\left(A_{i}(x) \wedge B_{i}(y), C_{i}(z)\right) .
\end{aligned}
$$

Noting Theorem 2.1 and according to Triple I algorithm, we obtain an inference result $C \in \mathcal{F}(Z)$ for arbitrary given $A \in \mathcal{F}(X), B \in \mathcal{F}(Y)$ :

$$
\begin{aligned}
& C(z)=\sup _{(x, y) \in X \times Y}\{A(x) \wedge B(y) \wedge[(\tilde{R}(x, y, z) \\
& -\lambda(1-A(x) \wedge B(y))) \vee 0]\}, z \in Z .
\end{aligned}
$$

For a input concrete vector $\left(x^{\prime}, y^{\prime}\right) \in X \times Y$, it is fuzzified by the following way

$$
A^{\prime}(x)=\left\{\begin{array}{l}
1, x=x^{\prime}, \\
0, x \neq x^{\prime},
\end{array} \quad B^{\prime}(y)=\left\{\begin{array}{l}
1, y=y^{\prime}, \\
0, y \neq y^{\prime} .
\end{array}\right.\right.
$$

Substituting it into (17), we obtain

$C^{\prime}(z)=\bigvee_{i=1}^{n} R_{L-\lambda-G}\left(A_{i}\left(x^{\prime}\right) \wedge B_{i}\left(y^{\prime}\right), C_{i}(z)\right), z \in Z$.

Since $C^{\prime}$ is a fuzzy set, a defuzzify-method is used to obtain the accurate scalar $z^{\prime} \in Z$. If $\int_{Z}|z| C^{\prime}(z) d z<+\infty$ and $0<\int_{Z} C^{\prime}(z) d z<+\infty$, then center of gravity defuzzifier is often used to obtain $z^{\prime}$. i.e.

$$
z^{\prime}=\frac{\int_{Z} z C^{\prime}(z) d z}{\int_{Z} C^{\prime}(z) d z} .
$$

Similar to (14), it follows that

$$
\begin{array}{ll}
\bar{s}: \quad & X \times Y \rightarrow Z \\
& (x, y) \mapsto \bar{s}(x, y) \\
& \triangleq \int_{Z} z C^{\prime}(z) d z / \int_{Z} C^{\prime}(z) d z .
\end{array}
$$

Then

$$
\begin{gathered}
z^{\prime}=\frac{\int_{Z} z C^{\prime}(z) d z}{\int_{Z} C^{\prime}(z) d z} \approx \frac{\sum_{i=1}^{n} z_{i} C^{\prime}\left(z_{i}\right) h_{i}}{\sum_{i=1}^{n} C^{\prime}\left(z_{i}\right) h_{i}} \\
=\frac{\sum_{i=1}^{n} z_{i}\left[\bigvee_{k=1}^{n} R_{L-\lambda-G}\left(A_{k}\left(x^{\prime}\right) \wedge B_{k}\left(y^{\prime}\right), C_{k}\left(z_{i}\right)\right)\right] h_{i}}{\sum_{i=1}^{n}\left[\bigvee_{k=1}^{n} R_{L-\lambda-G}\left(A_{k}\left(x^{\prime}\right) \wedge B_{k}\left(y^{\prime}\right), C_{k}\left(z_{i}\right)\right] h_{i}\right.} \\
=\frac{1}{z_{n}-e} \sum_{i=1}^{n} h_{i} z_{i}=\text { const. }
\end{gathered}
$$

If we choose $F(x, y)=\frac{1}{z_{n}-e} \sum_{i=1}^{n} h_{i} z_{i}$, then the theorem is proved.
Remark 2 Since $F(x, y)=$ const, this fuzzy system has not interpolation property. Thus the fuzzy system can almost be unapplied in the practical fuzzy control system.

Remark 3 Formula (16), which denotes a interface rule base, is a customary notation in engineering. The precise statement is seen in the reference [3] on the remark about completeness of rule.

Remark 4 "approximates to" in Theorem 3.1 and Theorem 3.2 means a limit of sum, which is regarded as integral. Indeed, greater $n$ is, and higher approximation is.

\section{Probability expression of fuzzy systems with a fam- ily of implication operators $L-\lambda-G$ based on Triple I} algorithm

On the base of [9], we shall discuss probability expression of fuzzy systems with a family of implication operators $L-\lambda-G$ based on Triple I algorithm.

Firstly, we consider the case of SISO. Noting that $B^{\prime}(y)$ in $(13)$ is related with $x^{\prime}$. Therefore $B^{\prime}(y)$ can be represented by

$$
B^{\prime}\left(y \mid x=x^{\prime}\right) \triangleq B^{\prime}(y),
$$

which means that a rough output $B^{\prime} \in \mathcal{F}(Y)$ is obtained under the condition $x=x^{\prime}$. Due to arbitrariness of $x^{\prime} \in X$, we directly rewrite $B^{\prime}\left(y \mid x=x^{\prime}\right)$ into the following form about a binary function $p: X \times Y \rightarrow \mathbb{R}:$

$$
p(x, y) \triangleq B^{\prime}\left(y \mid x=x^{\prime}\right)=B^{\prime}(y) .
$$

Thus formula (14) becomes

$$
\bar{s}(x)=\int_{Y} y p(x, y) d y / \int_{Y} p(x, y) d y .
$$

Function $p(x, y)$ is prolonged to $\mathbb{R}^{2}$ and defined by $q(x, y)$ :

$$
\begin{aligned}
q(x, y) & \triangleq p(x, y) I_{X \times Y}(x, y) \\
& \triangleq \begin{cases}p(x, y), & (x, y) \in X \times Y, \\
0, & (x, y) \notin X \times Y,\end{cases}
\end{aligned}
$$

where $I_{X \times Y}(x, y)$ is a characteristic function of $X \times$ $Y$. If $p(x, y)$ in $(22)$ is replaced with $q(x, y)$, then $\bar{s}(x)$ in $(22)$ is equivalent to a prolonged function in $\mathbb{R}$. For simplification, the prolonged function is still 
denoted by $\bar{s}(x)$. The same assumption is made in the following similar case. Thus (22) becomes

$$
\bar{s}(x)=\int_{-\infty}^{+\infty} y q(x, y) d y / \int_{-\infty}^{+\infty} q(x, y) d y .
$$

Further more, set

$H\left(2, n, R_{L-\lambda-G}, \vee\right) \triangleq \int_{-\infty}^{+\infty} \int_{-\infty}^{+\infty} q(x, y) d x d y$

$H\left(2, n, R_{L-\lambda-G}, \vee\right)$ is called a $H$ function with the parameters $\left(2, n, R_{L-\lambda-G}, \mathrm{~V}\right)$, where 2 denotes the binary function, parameter $n$ denotes the rule number used in inference rule (6) which constructs $q(x, y)$, parameter $R_{L-\lambda-G}$ denotes a fuzzy implication operator, parameter $V$ denotes a kind of residual triangle module used in synthesis relationship.

The aim of importing the $H$ function is only in order to reduction to unity. If $H\left(2, n, R_{L-\lambda-G}, \mathrm{~V}\right)>0$, then set

$$
f(x, y) \triangleq q(x, y) / H\left(2, n, R_{L-\lambda-G}, \vee\right) .
$$

It is obvious to see that (24) becomes

$$
\bar{s}(x)=\int_{-\infty}^{+\infty} y f(x, y) d y / \int_{-\infty}^{+\infty} f(x, y) d y .
$$

Theorem 4.1 The denotations on the fuzzy system of SISO implication operator $L-\lambda-G$ based on Triple I algorithm are same as above. If $\int_{Y}|y| p(x, y) d y<+\infty$ and $0<\int_{Y} p(x, y) d y<+\infty$, there exists a probability space $(\Omega, \mathcal{F}, P)$ and a random vector $(\xi, \eta)$ defined in this space such that

$$
E(\eta \mid \xi=x)=\bar{s}(x),
$$

which means that the value $\bar{s}(x)$ of function $\bar{s}$ at $x$ is equal to conditional mathematical expectation $E(\eta \mid \xi=x)$ of random variable $\eta$ under the condition $\xi=x$.

Proof. Let input universe and output universe be basic sets. We construct two probability space $\left(X, \mathcal{B}_{1}, P_{1}\right)$ and $\left(Y, \mathcal{B}_{2}, P_{2}\right)$, where $\mathcal{B}_{1}$ and $\mathcal{B}_{2}$ are Borel $\sigma$ - fields of $X$ and $Y$ respectively, then $P_{1}$ and $P_{2}$ are probability measures in $\mathcal{B}_{1}$ and $\mathcal{B}_{2}$ respectively. Suppose that $\xi$ and $\eta$ are random vectors respectively defined on $X$ and $Y$. Choose $\Omega \triangleq X \times Y, \mathcal{F} \triangleq \mathcal{B}_{1} \times \mathcal{B}_{2}$, and $P \triangleq P_{1} \times P_{2}$, where $\mathcal{F}$ is a Borel $\sigma$ - fields generated by cartesian product of two Borel $\sigma$ - fields $\mathcal{B}_{1}$ and $\mathcal{B}_{2}$. As well-known, $P$ is a product probability measure. Thus we obtain the joint probability space $(\Omega, \mathcal{F}, P)$. Without changing denotatins, define again the random variables $\xi$ and $\eta$ on $\Omega$ by the following way:

$$
\begin{array}{rlrl}
\xi: \Omega \rightarrow \mathbb{R}, & (x, y) & \mapsto \xi(x, y) \triangleq \xi(x), \\
\eta: \Omega \rightarrow \mathbb{R}, & (x, y) \mapsto \eta(x, y) \triangleq \eta(y) .
\end{array}
$$

Then, $(\xi, \eta)$ becomes a two-dimensional vector in joint probability space. According to the given conditions, a function defined by (26)

$$
\begin{array}{r}
f(x, y) \triangleq q(x, y) / H\left(2, n, R_{L-\lambda-G}, \vee\right) \\
=\frac{\left[\bigvee_{i=1}^{n} R_{L-\lambda-G}\left(A_{i}(x), B_{i}(y)\right)\right] I_{X \times Y}(x, y)}{\int_{X} \int_{Y}\left[\bigvee_{i=1}^{n} R_{L-\lambda-G}\left(A_{i}(x), B_{i}(y)\right)\right] d x d y},
\end{array}
$$

satisfies the condition of probability density function of two-dimensional random vector. Thus, take $f(x, y)$ as the probability density function of random vector $(\xi, \eta)$. From the definition of conditional mathematical expectation, in case of $\xi=x$ the conditional mathematical expectation of random variable $\eta$ in probability space $(\Omega, \mathcal{F}, P)$ is represented by

$$
E(\eta \mid \xi=x)=\int_{-\infty}^{+\infty} y f(x, y) d y / \int_{-\infty}^{+\infty} f(x, y) d y
$$

Hence, we have $E(\eta \mid \xi=x)=\bar{s}(x)$.

Next, we consider the case of double input and single output. Obviously, $C^{\prime}(z)$ in (18) is correlative to $\left(x^{\prime}, y^{\prime}\right)$, which is taken as $C^{\prime}(z) \triangleq C^{\prime}(z \mid x=$ $\left.x^{\prime}, y=y^{\prime}\right)$ or equal to the form of a triple function: $p: X \times Y \times Z \rightarrow \mathbb{R}$, where

$$
p(x, y, z) \triangleq C^{\prime}(z) .
$$

Thus, (18) becomes to

$$
\bar{s}(x, y)=\int_{Z} z p(x, y, z) d z / \int_{Z} p(x, y, z) d z .
$$

Similar to $(23), \quad p$ is prolonged to $\mathbb{R}^{3}$. Let $q(x, y, z) \triangleq p(x, y, z) I_{X \times Y \times Z}(x, y, z)$ and $(30)$ is translated into

$$
\begin{gathered}
\bar{s}(x, y)= \\
\int_{-\infty}^{+\infty} z q(x, y, z) d z / \int_{-\infty}^{+\infty} q(x, y, z) d z .
\end{gathered}
$$

In order to reduction to unity, set

$$
\begin{aligned}
& H\left(3, n, R_{L-\lambda-G}, \vee\right) \\
\triangleq & \int_{X} \int_{Y} \int_{Z} p(x, y, z) d x d y d z .
\end{aligned}
$$




$$
f(x, y, z) \triangleq q(x, y, z) / H\left(3, n, R_{L-\lambda-G}, \vee\right) .
$$

In accordance to proceding declaration, $\bar{s}(x, y)$ is equal to $\bar{s}(x, y) I_{X \times Y}(x, y)$. By $(31)$ we have

$$
\begin{gathered}
\bar{s}(x, y)= \\
\int_{-\infty}^{+\infty} z f(x, y, z) d z / \int_{-\infty}^{+\infty} f(x, y, z) d z .
\end{gathered}
$$

Theorem 4.2 The fuzzy system of fuzzy implication operator $L-\lambda-G$ with double input and single output is based on the Triple I algorithm. Its denotations concerned are stated above. If $\int_{Z}|z| p(x, y, z) d z<+\infty$ and $0<\int_{Z} p(x, y, z) d z<$ $+\infty$, then there exists a random vector $(\xi, \eta, \zeta)$ defined on a probability space $(\Omega, \mathcal{F}, P)$ such that

$$
E(\zeta \mid \xi=x, \eta=y)=\bar{s}(x, y) .
$$

Namely, the value $\bar{s}(x, y)$ of the function of $\bar{s}$ at $(x, y)$ is the conditional mathematical expectation $E(\zeta \mid \xi=x, \eta=y)$ of random variable $\zeta$ under the condition of $(\xi, \eta)=(x, y)$.

Proof. Assume that $X$ and $Y$ are input universes, and $Z$ is an output universe. We construct three probability spaces as

$$
\left(X, \mathcal{B}_{1}, P_{1}\right),\left(Y, \mathcal{B}_{2}, P_{2}\right),\left(Z, \mathcal{B}_{3}, P_{3}\right),
$$

where $\mathcal{B}_{1}, \mathcal{B}_{2}$ and $\mathcal{B}_{3}$ are Borel $\sigma$ - fields in $X, Y$ and $Z$ respectively, $P_{1}, P_{2}$ and $P_{3}$ are probability measures in $\mathcal{B}_{1}, \mathcal{B}_{2}$ and $\mathcal{B}_{3}$ respectively. Suppose that $\xi \eta$, and $\zeta$ are random variables defined by $X, Y$ and $Z$ respectively. Take $\Omega \triangleq X \times Y \times Z$, $P \triangleq \mathcal{B}_{1} \times \mathcal{B}_{2} \times \mathcal{B}_{3}$, and $\mathcal{F} \triangleq P_{1} \times P_{2} \times P_{3}$. We get joint probability space $(\Omega, \mathcal{F}, P)$. Without changing notations, renew $\xi, \eta$, and $\zeta$ defined in $\Omega$

$$
\begin{array}{lll}
\xi: \Omega \rightarrow \mathbb{R}, & (x, y, z) \mapsto \xi(x, y, z) \triangleq \xi(x), \\
\eta: \Omega \rightarrow \mathbb{R}, & (x, y, z) \mapsto \eta(x, y, z) \triangleq \eta(y), \\
\zeta: \Omega \rightarrow \mathbb{R}, & (x, y, z) \mapsto \zeta(x, y, z) \triangleq \zeta(z) .
\end{array}
$$

Then $\xi, \eta, \zeta$ becomes to be a three-dimensional random vector in $(\Omega, \mathcal{F}, P)$. According to the given conditions, the function defined by (33)

$$
\begin{gathered}
f(x, y, z) \triangleq q(x, y, z) / H\left(3, n, R_{L-\lambda-G}, \vee\right)= \\
\frac{\left[{ }_{i=1}^{n} R_{L-\lambda-G}\left(A_{i}(x) \wedge B_{i}(y), C_{i}(z)\right)\right] I_{X \times Y}(x, y, z)}{\int_{X} \int_{Y} \int_{Z}\left[{ }_{i=1}^{\vee} R_{L-\lambda-G}\left(A_{i}(x) \wedge B_{i}(y), C_{i}(z)\right)\right] d x d y d z}
\end{gathered}
$$

satisfies the condition of probability density function of three-dimensional random vector. Thus we take $f(x, y, z)$ as the probability density function of random vector $(\xi, \eta, \zeta)$. From the definition of conditional mathematical expectation, in case of $(\xi, \eta)=(x, y)$ the conditional mathematical expectation of random variable $\zeta$ in probability space $(\Omega, \mathcal{F}, P)$ is represented by

$$
\begin{aligned}
& E(\zeta \mid \xi=x, \eta=y) \\
= & \int_{-\infty}^{+\infty} z f(x, y, z) d z / \int_{-\infty}^{+\infty} f(x, y, z) d z .
\end{aligned}
$$

Hence, we have $E(\zeta \mid \xi=x, \eta=y)=\bar{s}(x, y)$.

Remark 5 By Theorem 4.1 and Theorem 4.2, we can construct a marginal density function, a conditional probability density function, a probability distribution function and marginal probability distribution function etc. from probability density function.

Remark 6 By (28) and (35), it follows two meanings as follows: (i) From the aspect of probability, probability density function $f(x, y)$ or $f(x, y, z)$ is a kernel of fuzzy systems with a family of implication operators $L-\lambda-G$, plays a "system kernel"role. It shows that it is essential that a fuzzy system with a family of implication operators $L-\lambda-G$ is a stochastic system. Consequently, for a fuzzy system, if probability density function $f(x, y)$ or $f(x, y, z)$ of a random variable $(\xi, \eta)$ or $(\xi, \eta, \eta)$ is obtained by means of probabilistic and statistical principle, then $\bar{s}(x)=E(\eta \mid \xi=x)$ or $\bar{s}(x, y)=E(\zeta \mid \xi=x, \eta=y)$ can be got. That is to say that we comprehend this system. (ii) From the construction process of fuzzy systems with a family of implication operators $L-\lambda-G$, the probability distribution of a stochastic system is determined by (6) (14).

\section{References}

[1] L.A. Zadeh, Outline of new approach to the analysis of complex systems and decision processes. IEEE Trans System, Man and Cybernetics, 3 (1): 28-33, 1973.

[2] G.J. Wang, The total implication Triple I algorithm on fuzzy inference. Science in China (Series E)(in Chinese), 29(1): 43-53, 1999.

[3] H.X. Li, Interpolation mechanism of fuzzy control. Science in China (Series E)(in Chinese), 28(3): 259-267, 1998. 
[4] H.X. Li, J.Y. Peng, J.Y. Wang, Fuzzy systems and their response functions based on commonly used fuzzy implication operators. Journal of Control Theory and Applications(in Chinese), 22(3): 341-347, 2005.

[5] H.X. Li, J.Y. Peng, J.Y. Wang, J. Hou, Y.Z. Zhang, Fuzzy systems based on Triple I algorithm and their response ability. Journal of Systems Science and Mathematical Science(in Chinese), 26(5): 578-590, 2006.

[6] G.J. Wang, R. Lan, Generalized tautologies of the systems $H_{\alpha}$. Journal of Shaanxi Normal University(Natural Science Edition)(in Chinese), 31(2): 1-11, 2003.

[7] W.M. Wu, Generalized tautologies in parametric Kleene's systems. Fuzzy Systems and Mathematics(in Chinese), 14(1): 1-7, 2000.

[8] X.F. Zhang, G.W. Meng, A. Zhang, Families of implication operators and their applications. Chinese Joural of Computers(in Chinese), 30(3): 448-453,2007.

[9] H.X. Li, The probibality experssion of fuzzy systems. Science in China (Series E)(in Chinese), 36(4): 373-397, 2006. 